\title{
The immunology of low-grade gliomas
}

\author{
Alexander F. Haddad, MD, Jacob S. Young, MD, Jun Yeop Oh, BS, Hideho Okada, MD, PhD, and \\ Manish K. Aghi, MD, PhD
}

Department of Neurological Surgery, University of California, San Francisco, California

\begin{abstract}
Low-grade gliomas (LGGs), which harbor an isocitrate dehydrogenase (IDH) mutation, have a better prognosis than their high-grade counterparts; nonetheless, they remain incurable and impart significant negative impacts on patients' quality of life. Although immunotherapies represent a novel avenue of treatment for patients with LGGs, they have not yet been successful. Accurately selecting and evaluating immunotherapies requires a detailed understanding of LGG tumor immunology and the underlying tumor immune phenotype. A growing body of literature suggests that LGGs significantly differ in their immunology from high-grade gliomas, highlighting the importance of investigation into LGG immunology specifically. In this review, the authors aimed to discuss relevant research surrounding the LGG tumor immune microenvironment, including immune cell infiltration, tumor immunogenicity, checkpoint molecule expression, the impact of an IDH mutation, and implications for immunotherapies, while also briefly touching on current immunotherapy trials and future directions for LGG immunology research.
\end{abstract}

https://thejns.org/doi/abs/10.3171/2021.11.FOCUS21587

KEYWORDS low-grade glioma; immunology; microenvironment; immune cell infiltration

$\mathrm{L}$ OW-GRADE gliomas (LGGs) are slow-growing glial tumors with the potential for malignant transformation. Their incidence is highest in patients in their 3rd and 4th decades of life, and both the tumor and the treatments can significantly affect the quality of life of patients. Seizure is the most common presenting symptom in the majority of patients, although headache and focal neurological deficits are also frequently seen. ${ }^{1,2}$ Indeed, LGGs are more often present in highly functional areas of the brain, with the insula and supplemental motor area being the two most commonly afflicted locations. ${ }^{3,4}$ The standard of care for LGGs includes surgical intervention, with the goal of providing maximal safe resection and acquisition of adequate tissue for detailed molecular and genetic characterization. ${ }^{5}$ This is becoming increasingly important, as the molecular subtypes of LGGs and their unique characteristics are becoming better defined. ${ }^{6}$

The utility of molecular and genetic characterization for LGGs is most evident in the latest 2021 WHO guidelines, which place an increased emphasis on molecular diagnostics. ${ }^{6}$ These updated guidelines for gliomas focus specifically on mutations in the isocitrate dehydrogenase 1
$(I D H 1)$ and $I D H 2$ genes, which have been associated with significantly improved outcomes in patients. ${ }^{7.8}$ As a result, adult LGG is now, by definition, IDH mutant, including both astrocytoma and oligodendroglioma (which also harbor a $1 \mathrm{p} / 19 \mathrm{q}$-codeletion). ${ }^{6}$ This is partly due to the differences in the natural history and outcomes of patients with IDH-wild-type and IDH-mutant gliomas. ${ }^{8}$

Few effective new therapeutic strategies for gliomas have been developed in the past decade. Immunotherapy, which targets the immune system against tumor cells, has been successful in other cancer types and is a major area of research for gliomas, albeit predominantly for highgrade gliomas (HGGs). Immunotherapies that are under investigation for gliomas include chimeric antigen receptor (CAR) T cells, systemically delivered checkpoint inhibitors (CPIs), oncolytic viral therapies, other viral gene therapies, and cancer vaccines, all of which have unique limitations. ${ }^{9}$ While previously thought to be immune-privileged, the brain has been highlighted in recent studies as being accessible to the immune system, suggesting that immunotherapies may hold promise in treating CNS tumors, including glioblastoma. ${ }^{10}$ Although initial trials of immunotherapies

ABBREVIATIONS AhR = aryl hydrocarbon receptor; $\mathrm{CAR}=$ chimeric antigen receptor; $\mathrm{CPI}=$ checkpoint inhibitor; $\mathrm{HGG}$ = high-grade glioma; IDH = isocitrate dehydrogenase; $L G G=$ low-grade glioma; MDM = monocyte-derived macrophage; MDSC = myeloid-derived suppressor cell; PD-1 = programmed cell death protein 1; PD-L1 = programmed death-ligand 1 ; poly-ICLC = polyinosinic-polycytidylic acid stabilized by lysine, and carboxymethylcellulose; TAM = tumor-associated macrophage; TDO = tryptophan 2,3-dioxygenase; TEX = tumor-derived glioma small extracellular vesicles; Treg = regulatory $\mathrm{T}$ cell; $2 \mathrm{HG}=\mathrm{R}$-2-hydroxyglutarate.

SUBMITTED October 1, 2021. ACCEPTED November 16, 2021.

INCLUDE WHEN CITING DOI: 10.3171/2021.11.FOCUS21587. 
in HGGs have been met with limited success, ${ }^{11,12}$ literature supporting different immunology in LGGs, and the hypothesis that altering the immune environment is a gradual process that might be more effective for LGGs than HGGs, have led to excitement about immunotherapies for treatment of LGGs. In this review, we summarize the immune phenotypes of patients with LGGs and how this may impact immunotherapies for LGGs as well as challenges and future directions in LGG immunology.

\section{Immunology of LGGs Immune Cell Infiltration}

Immune cell infiltration of tumors has been shown to play a role in tumor progression and has been used for cancer prognostication. ${ }^{13-15}$ Immune cell infiltration can also provide insight into how to best orchestrate a robust antitumor immune response using immunotherapy. Assessment of immune cell infiltration can be done using a variety of techniques including bulk and single-cell RNA sequencing, bulk genomic DNA samples, flow cytometry, cytometry by time of flight, immunofluorescence, and additional methodologies.

In a study of microarray and bulk RNA sequencing data from 2249 gliomas, Zhang et al. demonstrated that reduced tumor purity (i.e., increased invasion of a glioma by nontumor cells such as immune and stromal cells) was correlated with increased malignancy and reduced survival. ${ }^{16}$ The authors also showed that tumors with reduced glioma purity had increased immune cell scores, especially macrophage and neutrophil populations, relative to higher purity samples. Similarly, Aran et al., in an analysis of multiple cancer types, demonstrated an inverse relationship between tumor purity and mutational burden, with LGGs as one of the lowest mutational burdens and highest tumor purities in their analysis. ${ }^{17}$ These findings on tumor purity suggest that LGGs are relatively immune and stromal cell sparse relative to HGGs and other cancer types.

\section{T-Cell Compartment}

More detailed analyses of the LGG immune phenotype have shed additional light on specific immune cell infiltration. A study by Berghoff et al., which focused on the T-cell compartment in IDH-mutant and IDH-wild-type gliomas using immunohistochemistry, found that tumorinfiltrating lymphocytes and programmed death-ligand 1 (PD-L1) expression were significantly lower in IDHmutant gliomas compared with IDH-wild-type tumors. ${ }^{18}$ The authors also found that LGGs had significantly higher levels of PD-L1 methylation, likely explaining the reduced expression. Additional studies by Klemm et al. and Friebel et al. leveraged multifaceted approaches to assessing the CNS tumor microenvironment and similarly demonstrated low tumor-infiltrating lymphocyte levels in LGG relative to glioblastoma and brain metastasis. ${ }^{19,20}$ These findings suggest that LGGs, HGGs, and brain metastases lie on a spectrum of immunogenicity and T-cell infiltration, with brain metastases demonstrating the most T-cell-inflamed immune phenotype and LGGs the least-inflamed phenotype, with HGGs falling in between the two. Interestingly, similar trends have also been seen in the context of regu- latory $\mathrm{T}$ cells (Tregs), whereby Treg infiltration positively correlated with the glioma tumor grade. ${ }^{20,21}$ Friebel et al. also showed higher Treg infiltration in brain metastasis compared with HGGs and LGGs, similar to the trend seen with $\mathrm{T}$ cells in general. The differences in T-cell infiltration between LGGs, HGGs, and brain metastases suggest a tumor-intrinsic process driving the surrounding immune phenotype, rather than just the location intracranially.

The underlying biology of the differences in the T-cell compartment between various CNS tumors, especially IDH-mutant and IDH-wild-type gliomas, is an area of active research. Chongsathidkiet et al. implicated tumorimposed loss of S1P1 from the T-cell surface in sequestration of $\mathrm{T}$ cells in the bone marrow in multiple intracranial murine tumor models, including both glioma and metastasis, suggesting a common immunosuppressive pathway for brain tumors. ${ }^{22}$ Kohanbash et al., however, sought to investigate the specific role of IDH mutations in shaping the tumor microenvironment to further understand the unique immunophenotypes in IDH-mutant and IDHwild-type gliomas. ${ }^{23}$ Using The Cancer Genome Atlas (TCGA), the authors found reduced expression of T-cell lymphocyte-associated genes as well as CXCL10, which is implicated in the trafficking of CD8 T cells into brain tumor sites, in IDH-mutant tumors compared with IDHwild-type tumors. They then introduced mutant IDH1 or the downstream metabolite of mutant IDH, oncometabolite $R$-2-hydroxyglutarate ( $2 \mathrm{HG}$ ), to syngeneic mouse glioma models. Expression of $2 \mathrm{HG}$ or expression of mutant IDH1 reduced CXCL10 levels secondary to decreased production of STAT1 (a CXCL10 regulator); this was also associated with reduced T-cell infiltration in implanted tumors. Finally, IDH-C35, an inhibitor of mutant IDH1, reversed the immunophenotype seen in mutant tumors and increased the efficacy of vaccine immunotherapy. ${ }^{23}$ These findings implicated the production of $2 \mathrm{HG}$ by mutant IDH1 in molding the eventual immunophenotype seen in these tumors. Interestingly, mutant IDH does not seem to have as strong of an effect on CD4 T-cell levels, although previous research has demonstrated that CD4 T cells are, in fact, capable of generating an immune response against the R123H IDH mutation. ${ }^{24,25}$

\section{Myeloid Cell Compartment}

While $\mathrm{T}$ cells play an undeniable role in generating a robust antitumor immune response, significant consideration should also be paid to the myeloid compartment of a tumor's immune phenotype. Myeloid cells, which make up a much larger percentage of the immune cell compartment relative to T cells, can play a range of roles in supporting or hampering an antitumor immune response, and differences in the infiltration of myeloid cells can have an important impact on the efficacy of immunotherapies (Fig. 1). Key classes of myeloid cells in the tumor microenvironment include dendritic cells, neutrophils, tumor-associated macrophages (TAMs; including monocyte-derived macrophages [MDMs] and microglia), and myeloid-derived suppressor cells (MDSCs). ${ }^{26-28}$ Indeed, TAMs comprise the majority of IDH-mutant and IDHwild-type tumors, significantly outnumbering the number of other immune cell types, including T cells. ${ }^{19,20}$ Interest- 


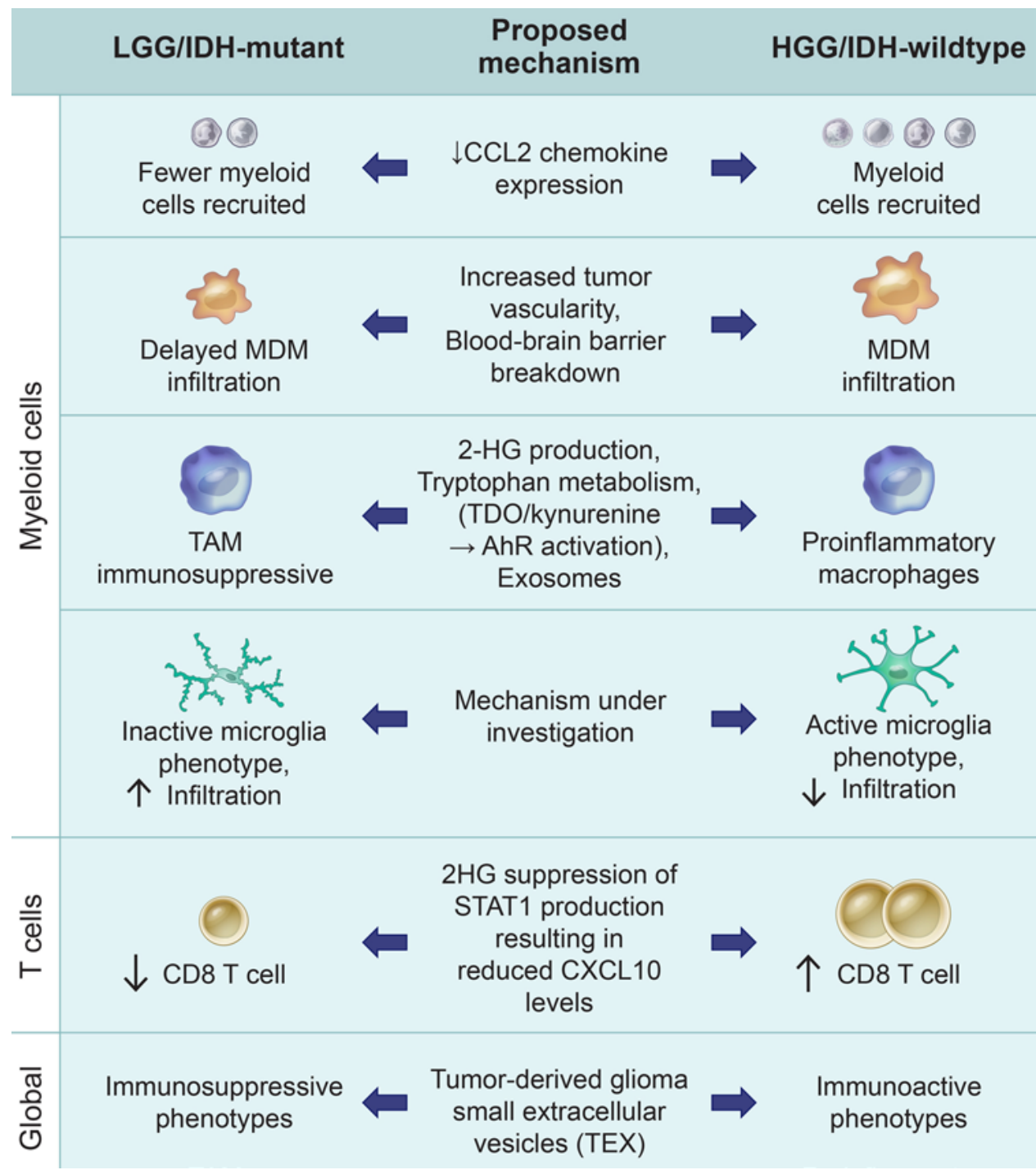

FIG. 1. Schematic summarizing differences in the immunology of IDH-mutant LGGs and IDH-wild-type HGGs as well as proposed associated mechanisms. $\uparrow=$ increased; $\downarrow=$ decreased. @ Noel Sirivansanti, published with permission.

ingly, the dominant type of TAM differs between IDHmutant and wild-type tumors, with IDH-mutant TAMs being composed primarily of microglia and IDH-wild-type tumors (and brain metastases) associated with primarily MDMs. ${ }^{19,20,29}$ The microglia present in IDH-wild-type tumors and brain metastases also appear to be more of an activated phenotype than those found in IDH-mutant tumors, as determined by differences in CDI4 and CD64 genes and cell morphology. ${ }^{20}$ Within LGGs, Müller et al. demonstrated significantly reduced infiltrating microglia in oligodendrogliomas relative to astrocytomas; LGGs with higher MDM infiltration also had significantly reduced survival. ${ }^{29}$ The difference in microglial and MDM cells in IDH-mutant astrocytomas and oligodendrogliomas was also noted by Venteicher et al. ${ }^{30}$ In their study, astrocytomas also demonstrated increased MDMs as the tumor grade increased, which corresponded with an increase in the expression of endothelial-specific genes. The association between MDMs and endothelial genes may be due to increased access of MDMs to the tumor microenvironment from systemic circulation. It is also important to note that tumor-infiltrating MDMs lie on a spectrum; Venteicher et al. noted that MDMs in the glioma microenvironment expressed less macrophage-specific genes than those found in brain metastasis, suggesting a range of macrophage states rather than clearly defined and circumscribed cellular states. ${ }^{30}$ Given the difficulty in distinguishing MDSCs from other myeloid subtypes in the tumor microenvironment, there is a paucity of data on MDSC infiltration in LGGs; they are likely being labeled as either MDMs or neutrophils (depending on if they are granulocytic or monocytic) in studies to date.

The intratumoral forces driving the patterns seen in myeloid cell infiltration in LGGs are still unclear. As previously discussed, the association between tumor grade, endothelial gene expression, and MDM infiltration noted by Venteicher et al. may suggest that increased exposure to the systemic circulation, through increased tumor vas- 
cularity and blood-brain barrier breakdown, may contribute to differences in myeloid infiltration between LGGs and HGGs. ${ }^{30}$ Increased MDMs in low-grade astrocytomas relative to oligodendrogliomas also potentially indicate a different tumor cell-intrinsic mechanism. Seeking to evaluate the effect of tumor genotype on the myeloid cell compartment, Friedrich et al. assessed alterations in the immune phenotype of tumors in response to IDH mutation using a variety of molecular tools. ${ }^{31}$ Similar to the aforementioned studies, the authors determined that microglia and macrophages demonstrated wide separation on principal component analysis corresponding with IDH status following RNA sequencing in HGGs. Using the GL261 mouse model of glioblastoma, they found that the myeloid compartment of the tumor microenvironment was significantly impacted by tumor IDH status. Mice implanted with IDH-mutant tumors demonstrated lower expression of the CCL2 chemokine (which recruits myeloid cells), less immunoactivated microglia, and delayed MDM infiltration relative to wild-type tumors. Interestingly, MDMs from IDH-mutant tumors showed an increased ability to suppress T-cell production of interferon- $\gamma$ (IFN $\gamma$ ) and incite expression of the programmed cell death protein 1 (PD-1) exhaustion marker. Inhibiting IDH led to a proinflammatory phenotype in macrophages, while the exogenously administered $2 \mathrm{HG}$ recapitulated the MDM (but not microglia) findings seen in IDH-mutant tumors, directly implicating $2 \mathrm{HG}$ in the immunosuppressive macrophage phenotypes seen in IDH-mutant tumors. The authors then further identified tryptophan metabolism, specifically the enzyme tryptophan 2,3-dioxygenase (TDO) and its product kynurenine, as playing a key role in aryl hydrocarbon receptor (AhR) activation and the downstream immunosuppressive effects previously characterized. ${ }^{31}$ These results provide some insight into how IDH-mutant tumors, which, by definition, are LGGs, suppress the surrounding immune microenvironment.

A recent study by Ludwig et al. identified an alternative pathway in which IDH-mutant gliomas can generate an immunosuppressive phenotype both locally and systemically through tumor-derived glioma small extracellular vesicles (TEX). ${ }^{32}$ The authors found that IDH-mutant gliomas produced larger, more immunosuppressive, and morphologically distinct TEX from IDH-wild-type tumors. Injection of IDH-mutant TEX into wild-type tumors led to reductions in tumor-infiltrating natural killer cells and increases in immunosuppressive Tregs and mono-MDSCs. Interestingly, injection of IDH-wild-type TEX into IDH-mutant tumors increased effector lymphocytes and M1 macrophages and reduced MDSCs. ${ }^{32}$ The studies by Ludwig et al. and Friedrich et al. highlight the still-developing theories on how IDH impacts glioma tumor immunology. ${ }^{31,32}$ Additional studies are required to fully understand the impact of IDH on immune cell infiltration, specifically in LGGs, and potential therapeutic vulnerabilities.

\section{Additional Aspects of LGG Immunogenicity \\ Antigen Presence and Presentation}

Tumor mutational burden is seen as a potential indicator of immunogenicity, as in many cancer types it is thought that an increased mutational burden is also likely indicative of the overall tumor antigen number, providing more "targets" for the immune system. ${ }^{33-37}$ This was demonstrated in a study by Samstein et al., who assessed the tumor mutational burden of 1662 patients with CPI treatment and 5371 without CPI. ${ }^{37}$ The authors found that, for most cancers, patients with a higher mutational burden had better responses to CPI treatment, despite inconsistent mutational cutoffs between cancer types. Interestingly, gliomas did not follow the same trend as other cancers, trending instead toward worse survival in those patients with high tumor mutational burdens. Alternatively, hypermutated gliomas have been shown to have low T-cell infiltration, high intratumoral heterogeneity, and a poor response to anti-PD-1 therapy. ${ }^{38}$ This may be due to temozolomide-induced mutations, which are frequently single-nucleotide variant mutations and do not adequately induce an antitumor immune response. In fact, in HGGs, low mutational burden has been associated with improved outcomes following viral or CPI therapy. HGGs with a lower mutational burden had increased inflammatory gene signals, suggesting that neoantigens were removed from the tumor via immunoediting. ${ }^{39}$ In LGGs, a higher mutational burden has similarly been associated with worse outcomes; ${ }^{40}$ this is especially true if patients develop temozolomide-related hypermutation upon transformation to a higher grade. ${ }^{41}$ How hypermutation specifically impacts the immune tumor microenvironment in LGGs is still not fully elucidated and warrants additional investigation.

Similarly, there is a paucity of literature on antigen presentation and dendritic cells in LGGs. Dendritic cells are critical for immune surveillance of the CNS. They can capture antigens, process them, and present them to T cells (both cytotoxic and helper) and B cells, thereby acting as a bridge between the innate and adaptive immune systems. The literature on dendritic cell infiltration in LGGs is limited, but there appear to be few dendritic cells in both HGG IDH-wild-type and LGG IDH-mutant tumors. ${ }^{19}$ Interestingly, it appears that LGG IDH-mutant tumors have a reduced capacity for antigen presentation, as IDH-wildtype tumors have increased infiltration of MDMs expressing major histocompatibility complex (MHC) class II human leukocyte antigen-DR isotype (HLA-DR). HGG IDH-wild-type MDMs also have increased expression of antigen presentation gene sets, antigen processing associated pathways, and MHC class I presentation gene sets. ${ }^{19}$ It must be noted, however, that HGG IDH-wild-type tumors remain resistant to immunotherapy and MDMs have been implicated in tumor progression, making it difficult to interpret their possibly increased ability for antigen presentation. Additional analysis is needed to compare the functional antigen presentation ability of MDMs from IDH-mutant tumors with those from IDH-wild-type tumors to better understand their ability and how to harness them for immunotherapy.

\section{Checkpoint Molecule Expression}

Checkpoint molecules are frequently pathologically coopted by cancers to avoid immune surveillance and killing. This has driven the development of monoclonal antibodies to block checkpoint molecules, also known as a 
CPI. While checkpoint molecule expression in the tumor microenvironment may not always correlate with tumor responsiveness to checkpoint inhibition, it is still important to consider. Garber et al. sought to assess PD-1 and PD-L1 expression in various glioma grades. ${ }^{42}$ The authors found that both PD-1-positive tumor-infiltrating lymphocytes and PD-L1 expression in tumor cells were correlated with increasing tumor grade..$^{42}$ Interestingly, there did not appear to be a relationship between IDH-mutant tumors and either PD-1-positive tumor-infiltrating lymphocytes or PD-L1-expressing tumor cells. ${ }^{42}$ A subsequent study by Berghoff et al. found significantly higher levels of PD-1-positive tumor-infiltrating lymphocytes and PDL1 expression in IDH-wild-type gliomas compared with IDH-mutant gliomas. ${ }^{43}$ They also found that IDH-mutant tumor cells had higher PD-L1 promoter methylation, perhaps explaining their observations. The findings by Berghoff et al. correlate with a study of 1024 glioma samples by Liu et al. that demonstrated higher CTLA-4 expression in higher-grade IDH-wild-type tumors compared with lower-grade IDH-mutant tumors. ${ }^{44}$ Overall, it appears that IDH-wild-type HGGs express higher levels of checkpoint molecules, potentially making them more attractive candidates for CPI immunotherapy. However, further investigation is needed to elucidate the underlying molecular biology informing the observed trends.

\section{Current Immunotherapy Trials for LGGs}

As discussed in this review article, LGGs have unique tumor immune phenotypes, with their IDH mutations potentially making them more or less vulnerable to specific types of immunotherapies. Compared with HGGs, there is a relative paucity of clinical trials evaluating immunotherapies for IDH-mutant LGGs. This is likely due to a variety of factors, including the better prognosis associated with LGGs and their lower incidence, resulting in the need for relatively longer clinical trials to evaluate the efficacy of a novel treatment. An additional difficulty in developing immunotherapies for LGGs is the differences in immune gene expression between HGGs and LGGs, which can hinder the ability for HGG immunotherapy trials to be translated to LGGs. For example, CAR T-cell targets for HGGs, such as EGFRvIII and IL13Ra2, are not found in LGGs, preventing their use in LGGs and requiring the identification of LGG-specific targets.

However, immunotherapeutic modalities, such as vaccines, may offer a safe and effective option for patients with LGGs due to the slower growth rate of the tumors (in contrast with HGGs), which should allow sufficient time for multiple immunizations and higher levels of antiglioma immunity. Furthermore, the generally mild toxicity of vaccines may have advantages over chemotherapy or radiation therapy for long-term cognitive and quality-of-life impairments. Okada et al. conducted the first vaccine trial that was specifically designed for patients with LGGs and reported that these patients, especially those who had not received chemotherapy or radiation therapy, demonstrated an excellent immunological response to the vaccine. ${ }^{45}$

Immunotherapies that are being evaluated in IDH-mutant LGGs now include CPIs; polyinosinic-polycytidylic acid stabilized by lysine, and carboxymethylcellulose
(poly-ICLC); cancer vaccines; and IDH-mutant inhibitors. A list of immunotherapy clinical trials including IDH-mutant LGGs and their underlying rationales can be found in Tables 1 and 2, respectively. Thus far, no immunotherapy has demonstrated a robust survival impact in clinical trials for LGGs, although the slow rate of progression in these gliomas means that very few trials have completed their evaluation (Table 1). Promising newer developments in immunotherapy for LGGs include the combination of IDH-mutant inhibitors with another immunotherapy such as a CPI. This may help to reduce the immune suppression associated with IDH-mutant tumors before adding an additional agent to stimulate the immune system. ${ }^{46}$

\section{Conclusions}

Despite their slow-growing nature, LGGs lead to significant morbidity and mortality. They are, in part, defined by the presence of mutant IDH, which may alter their underlying biology in a variety of ways compared with IDH-wild-type tumors. Recent studies using novel multifaceted immune-profiling approaches have significantly increased our understanding of LGGs, which have tumor immune microenvironments that are uniquely immunosuppressed compared with IDH-wild-type HGGs and brain metastases; LGGs have been shown to have reduced T-cell infiltration and decreased infiltration of myeloid cells with the potential for antigen presentation. Initial studies have demonstrated the importance of mutant IDH and its byproduct $2 \mathrm{HG}$ in mediating this unique immune phenotype, but a complete understanding of the molecular pathways implicated is lacking. As a result, significant investigation is needed to better understand the LGG immune microenvironment, underlying biological pathways, and implications for immunotherapies.

There are a number of areas within LGG research that warrant additional investigation and development. One such area of improvement that will allow for future advancement in the realm of immunology and immunotherapy for patients with LGGs is the generation of preclinical cell lines that are better biomimetic models for human LGG tumors. Currently, the majority of mouse glioma models are created in an effort to recapitulate glioblastoma rather than LGGs. ${ }^{47}$ Indeed, studies investigating how IDH mutation affects the immune microenvironment have primarily been performed in murine glioblastoma models. Introducing IDH mutations into murine glioblastoma cell lines likely does not accurately model the tumorigenesis and pathology of human LGGs, given the significant differences in tumor invasion and breakdown of the bloodbrain barrier, which may impact immune profiles. An additional difficulty with investigating immunotherapy for LGGs is the longer and variable patient survival associated with these tumors, which makes clinical trials logistically challenging and more expensive compared with glioblastoma trials. Finally, the systemic impact of gliomas on the immune system is better defined for HGGs than LGGs. ${ }^{22}$ Although our previous vaccine trial in patients with LGGs suggested that those patients can mount superior immune responses against peptide vaccines compared with patients with HGGs or pediatric patients with gliomas, ad- 
TABLE 1. Recruiting, currently active, completed, and terminated clinical trials examining immunotherapy or small molecules for LGGs

\begin{tabular}{|c|c|c|c|c|c|c|c|}
\hline $\begin{array}{l}\text { ClinicalTrials.gov } \\
\text { Identifier }\end{array}$ & $\begin{array}{l}\text { Experimental } \\
\text { Treatment }\end{array}$ & $\begin{array}{l}\text { Control/ } \\
\text { Comparator } \\
\text { Treatment }\end{array}$ & $\begin{array}{l}\text { No. } \\
\text { of } \\
\text { Pts }\end{array}$ & $\begin{array}{c}\text { Primary Endpoint/ } \\
\text { Outcomes }\end{array}$ & $\begin{array}{c}\text { Results for } \\
\text { Primary Outcome }\end{array}$ & $\begin{array}{l}\text { Study Start } \\
\text { Date }\end{array}$ & $\begin{array}{l}\text { Current } \\
\text { Status }\end{array}$ \\
\hline \multicolumn{8}{|l|}{ Phase I trials } \\
\hline NCT02924038 & $\begin{array}{l}\text { IMA950, } \\
\text { poly-ICLC, } \\
\text { varlilumab }\end{array}$ & None & 14 & $\begin{array}{l}\text { Incidence of } A E s \text {, } \\
\text { response rate of CD4+ } \\
\text { T-cell responses, mag- } \\
\text { nitude of CD8+ T-cell } \\
\text { responses }\end{array}$ & Not posted & $\begin{array}{l}\text { April 3, } \\
2017\end{array}$ & $\begin{array}{l}\text { Active, not } \\
\text { recruiting }\end{array}$ \\
\hline NCT03343197 & AG-120, AG881 & None & 49 & $\begin{array}{l}2 \mathrm{HG} \text { concentration in } \\
\text { resected tumors }\end{array}$ & Not posted & $\begin{array}{l}\text { March 20, } \\
2018\end{array}$ & $\begin{array}{l}\text { Active, not } \\
\text { recruiting }\end{array}$ \\
\hline NCT01130077 & $\begin{array}{l}\text { HLA-A2-restricted } \\
\text { glioma antigen } \\
\text { peptides vac- } \\
\text { cine, poly-ICLC }\end{array}$ & None & 60 & Safety & Not posted & $\begin{array}{c}\text { February } \\
2009\end{array}$ & $\begin{array}{l}\text { Active, not } \\
\text { recruiting }\end{array}$ \\
\hline NCT02549833 & $\begin{array}{l}\text { GBM6-AD, poly- } \\
\text { ICLC }\end{array}$ & None & 30 & $\begin{array}{l}\text { No. of regimen limiting } \\
\text { toxicity, measurement } \\
\text { of vaccine-induced im- } \\
\text { mune response in the } \\
\text { resected tumor }\end{array}$ & Not posted & $\begin{array}{l}\text { October 17, } \\
2016\end{array}$ & Recruiting \\
\hline \multicolumn{8}{|l|}{ Phase II trials } \\
\hline NCT04544007 & Poly-ICLC & None & 20 & Overall response rate & Not posted & $\begin{array}{r}\text { September } \\
30,2021\end{array}$ & $\begin{array}{l}\text { Not yet } \\
\text { recruiting }\end{array}$ \\
\hline NCT01188096 & Poly-ICLC & None & 23 & Overall response rate & $\begin{array}{l}\text { Preliminary analysis: FU range } \\
3-58 \text { mos, } 43 \% \text { stable disease, } \\
\quad \& 17 \% \text { partial responses }{ }^{49}\end{array}$ & $\begin{array}{r}\text { August } \\
2010\end{array}$ & Completed \\
\hline NCT01635283 & $\begin{array}{l}\text { Tumor lysated- } \\
\text { pulse autolo- } \\
\text { gous dendritic } \\
\text { cell vaccine }\end{array}$ & None & 5 & Progression-free survival & $\begin{array}{l}\text { Time w/o being affected by tumor } \\
\text { recurrence or progression: } \\
7-12 \text { mos }(n=1 / 5), 13-18 \text { mos } \\
(n=1 / 5), 19-24 \text { mos }(n=1 / 5) \\
>30 \operatorname{mos}(n=2 / 5)\end{array}$ & $\begin{array}{c}\text { May } 13, \\
2016\end{array}$ & Completed \\
\hline NCT03718767 & Nivolumab & None & 95 & $\begin{array}{l}\text { 6-mo progression-free } \\
\text { survival rate }\end{array}$ & Not posted & $\begin{array}{l}\text { March 27, } \\
2019\end{array}$ & Recruiting \\
\hline NCT04458272 & DS-1001b & None & 25 & $\begin{array}{l}\text { Overall response rate, } \\
\text { multiple pts w/ treat- } \\
\text { ment-emergent AEs }\end{array}$ & Not posted & $\begin{array}{l}\text { July } 8 \text {, } \\
2020\end{array}$ & $\begin{array}{l}\text { Active, not } \\
\text { recruiting }\end{array}$ \\
\hline
\end{tabular}

$\mathrm{AE}=$ adverse event; $\mathrm{FU}=$ follow-up; pts $=$ patients.

TABLE 2. Underlying rationale behind various immunotherapies for LGGs

\begin{tabular}{|c|c|}
\hline Therapy & Rationale \\
\hline $\begin{array}{l}\text { Systemically } \\
\text { delivered CPI }\end{array}$ & $\begin{array}{l}\text { Blocks inhibitory interactions w/ checkpoint molecules } \\
\text { \& allows for the induction or continuation of an } \\
\text { antitumor immune response }\end{array}$ \\
\hline Poly-ICLC & $\begin{array}{l}\text { Immunostimulatory molecule that leads to increased } \\
\text { proinflammatory gene expression \& attempts to } \\
\text { stimulate/boost an antitumor immune response }\end{array}$ \\
\hline $\begin{array}{l}\text { Cancer } \\
\text { vaccines }\end{array}$ & $\begin{array}{l}\text { Seek to train \& target the immune system against } \\
\text { tumor-associated antigens to stimulate an antitumo } \\
\text { immune response }\end{array}$ \\
\hline $\begin{array}{l}\text { IDH-mutant } \\
\text { inhibitors }\end{array}$ & $\begin{array}{l}\text { Variety of mechanisms; may contribute to increased } \\
\text { antitumor immune activity by reversing or limiting } \\
\text { IDH-mutant-associated immune suppression }\end{array}$ \\
\hline
\end{tabular}

ditional insight into how LGGs impact systemic immunity is needed. ${ }^{45}$ Whether or not LGGs lead to the T-cell sequestration similar to that seen in HGGs is unclear. This is especially relevant because patients with LGGs may be treated with temozolomide for long periods of time, which can also lead to systemic immune suppression and might interfere with certain immunotherapy treatments. ${ }^{48}$

\section{References}

1. Forst DA, Nahed BV, Loeffler JS, Batchelor TT. Low-grade gliomas. Oncologist. 2014;19(4):403-413.

2. Pouratian N, Asthagiri A, Jagannathan J, Shaffrey ME, Schiff D. Surgery Insight: the role of surgery in the management of low-grade gliomas. Nat Clin Pract Neurol. 2007; 3(11):628-639.

3. Duffau H, Capelle L. Preferential brain locations of lowgrade gliomas. Cancer. 2004;100(12):2622-2626.

4. Parisot S, Darlix A, Baumann C, et al. A probabilistic atlas 
of diffuse WHO Grade II glioma locations in the brain. PLoS One. 2016;11(1):e0144200.

5. Brown TJ, Bota DA, van Den Bent MJ, et al. Management of low-grade glioma: a systematic review and meta-analysis. Neurooncol Pract. 2019;6(4):249-258.

6. Louis DN, Perry A, Wesseling P, et al. The 2021 WHO Classification of Tumors of the Central Nervous System: a summary. Neuro Oncol. 2021;23(8):1231-1251.

7. Han S, Liu Y, Cai SJ, et al. IDH mutation in glioma: molecular mechanisms and potential therapeutic targets. $\mathrm{Br} \mathrm{J}$ Cancer. 2020;122(11):1580-1589.

8. Yan H, Parsons DW, Jin G, et al. IDH1 and IDH2 mutations in gliomas. N Engl J Med. 2009;360(8):765-773.

9. Lim M, Xia Y, Bettegowda C, Weller M. Current state of immunotherapy for glioblastoma. Nat Rev Clin Oncol. 2018; 15(7):422-442.

10. Louveau A, Smirnov I, Keyes TJ, et al. Structural and functional features of central nervous system lymphatic vessels. Nature. 2015;523(7560):337-341.

11. Young JS, Dayani F, Morshed RA, Okada H, Aghi MK. Immunotherapy for high grade gliomas: a clinical update and practical considerations for neurosurgeons. World Neurosurg. 2019;124:397-409.

12. Reardon DA, Brandes AA, Omuro A, et al. Effect of nivolumab vs bevacizumab in patients with recurrent glioblastoma: the CheckMate 143 phase 3 randomized clinical trial. JAMA Oncol. 2020;6(7):1003-1010.

13. Grivennikov SI, Greten FR, Karin M. Immunity, inflammation, and cancer. Cell. 2010;140(6):883-899.

14. Song LR, Weng JC, Li CB, et al. Prognostic and predictive value of an immune infiltration signature in diffuse lowergrade gliomas. JCI Insight. 2020;5(8):133811.

15. Haddad AF, Chen JS, Oh T, Pereira MP, Joshi RS, Aghi MK. Higher cytolytic score correlates with an immunosuppressive tumor microenvironment and reduced survival in glioblastoma. Sci Rep. 2020;10(1):17580.

16. Zhang C, Cheng W, Ren X, et al. Tumor purity as an underlying key factor in glioma. Clin Cancer Res. 2017;23(20):6279-6291.

17. Aran D, Sirota M, Butte AJ. Systematic pan-cancer analysis of tumour purity. Nat Commun. 2015;6:8971.

18. Berghoff AS, Kiesel B, Widhalm G, et al. Programmed death ligand 1 expression and tumor-infiltrating lymphocytes in glioblastoma. Neuro Oncol. 2015;17(8):1064-1075.

19. Klemm F, Maas RR, Bowman RL, et al. Interrogation of the microenvironmental landscape in brain tumors reveals disease-specific alterations of immune cells. Cell. 2020; 181(7):1643-1660.e17.

20. Friebel E, Kapolou K, Unger S, et al. Single-cell mapping of human brain cancer reveals tumor-specific instruction of tissue-invading leukocytes. Cell. 2020;181(7):1626-1642.e20.

21. Heimberger AB, Abou-Ghazal M, Reina-Ortiz C, et al. Incidence and prognostic impact of FoxP3+ regulatory T cells in human gliomas. Clin Cancer Res. 2008;14(16):5166-5172.

22. Chongsathidkiet P, Jackson C, Koyama S, et al. Sequestration of T cells in bone marrow in the setting of glioblastoma and other intracranial tumors. Nat Med. 2018;24(9):1459-1468.

23. Kohanbash G, Carrera DA, Shrivastav S, et al. Isocitrate dehydrogenase mutations suppress STAT1 and CD8+ T cell accumulation in gliomas. J Clin Invest. 2017;127(4):1425-1437.

24. Schumacher T, Bunse L, Pusch S, et al. A vaccine targeting mutant IDH1 induces antitumour immunity. Nature. 2014; 512(7514):324-327.

25. Pellegatta S, Valletta L, Corbetta $\mathrm{C}$, et al. Effective immunotargeting of the IDH1 mutation $\mathrm{R} 132 \mathrm{H}$ in a murine model of intracranial glioma. Acta Neuropathol Commun. 2015;3:4.

26. Pombo Antunes AR, Scheyltjens I, Lodi F, et al. Single-cell profiling of myeloid cells in glioblastoma across species and disease stage reveals macrophage competition and specialization. Nat Neurosci. 2021;24(4):595-610.
27. Mi Y, Guo N, Luan J, et al. the emerging role of myeloidderived suppressor cells in the glioma immune suppressive microenvironment. Front Immunol. 2020;11:737.

28. Andersen BM, Faust Akl C, Wheeler MA, Chiocca EA, Reardon DA, Quintana FJ. Glial and myeloid heterogeneity in the brain tumour microenvironment. Nat Rev Cancer. 2021; 21(12):786-802.

29. Müller S, Kohanbash G, Liu SJ, et al. Single-cell profiling of human gliomas reveals macrophage ontogeny as a basis for regional differences in macrophage activation in the tumor microenvironment. Genome Biol. 2017;18(1):234.

30. Venteicher AS, Tirosh I, Hebert C, et al. Decoupling genetics, lineages, and microenvironment in IDH-mutant gliomas by single-cell RNA-seq. Science. 2017;355(6332):eaai8478.

31. Friedrich M, Sankowski R, Bunse L, et al. Tryptophan metabolism drives dynamic immunosuppressive myeloid states in IDH-mutant gliomas. Nat Cancer. 2021;2(7):723-740.

32. Ludwig N, Rao A, Sandlesh P, et al. Characterization of systemic immunosuppression by IDH mutant glioma small extracellular vesicles. Neuro Oncol. Published online July 13, 2021. doi:10.1093/neuonc/noab153

33. Gubin MM, Schreiber RD. The odds of immunotherapy success. Science. 2015;350(6257):158-159.

34. Van Allen EM, Miao D, Schilling B, et al. Genomic correlates of response to CTLA-4 blockade in metastatic melanoma. Science. 2015;350(6257):207-211.

35. Valero C, Lee M, Hoen D, et al. The association between tumor mutational burden and prognosis is dependent on treatment context. Nat Genet. 2021;53(1):11-15.

36. Yarchoan M, Hopkins A, Jaffee EM. Tumor mutational burden and response rate to PD-1 inhibition. $N$ Engl J Med. 2017;377(25):2500-2501

37. Samstein RM, Lee CH, Shoushtari AN, et al. Tumor mutational load predicts survival after immunotherapy across multiple cancer types. Nat Genet. 2019;51(2):202-206.

38. Touat M, Li YY, Boynton AN, et al. Mechanisms and therapeutic implications of hypermutation in gliomas. Nature. 2020;580(7804):517-523.

39. Gromeier M, Brown MC, Zhang G, et al. Very low mutation burden is a feature of inflamed recurrent glioblastomas responsive to cancer immunotherapy. Nat Commun. 2021;12(1):352.

40. Alghamri MS, Thalla R, Avvari RP, et al. Tumor mutational burden predicts survival in patients with low-grade gliomas expressing mutated IDH1. Neurooncol Adv. 2020;2(1):a042.

41. Yu Y, Villanueva-Meyer J, Grimmer MR, et al. Temozolomideinduced hypermutation is associated with distant recurrence and reduced survival after high-grade transformation of low-grade IDH-mutant gliomas. Neuro Oncol. 2021;23(11):1872-1884.

42. Garber ST, Hashimoto Y, Weathers SP, et al. Immune checkpoint blockade as a potential therapeutic target: surveying CNS malignancies. Neuro Oncol. 2016;18(10):1357-1366.

43. Berghoff AS, Kiesel B, Widhalm G, et al. Correlation of immune phenotype with IDH mutation in diffuse glioma. Neuro Oncol. 2017;19(11):1460-1468.

44. Liu F, Huang J, Liu X, Cheng Q, Luo C, Liu Z. CTLA-4 correlates with immune and clinical characteristics of glioma. Cancer Cell Int. 2020;20:7.

45. Okada H, Butterfield LH, Hamilton RL, et al. Induction of robust type-I CD8+ T-cell responses in WHO grade 2 low-grade glioma patients receiving peptide-based vaccines in combination with poly-ICLC. Clin Cancer Res. 2015;21(2):286-294.

46. Friedrich M, Bunse L, Wick W, Platten M. Perspectives of immunotherapy in isocitrate dehydrogenase-mutant gliomas. Curr Opin Oncol. 2018;30(6):368-374.

47. Haddad AF, Young JS, Amara D, et al. Mouse models of glioblastoma for the evaluation of novel therapeutic strategies. Neurooncol Adv. 2021;3(1):b100.

48. Mathios D, Kim JE, Mangraviti A, et al. Anti-PD-1 antitumor immunity is enhanced by local and abrogated by systemic chemotherapy in GBM. Sci Transl Med. 2016;8(370):370ra180. 
49. Aguilera D, MacDonald T, Crawford J, et al. LGG-58. A Phase II trial of poly-ICLC in the management of recurrent or progressive pediatric low grade gliomas (NCT01188096): preliminary analysis. Neuro Oncol. 2018;20(2)(suppl):i116.

\section{Disclosures}

Dr. Okada: consultant for Bristol-Myers Squibb, Alexion Pharmaceuticals, Amal Therapeutics, Servier Pharmaceuticals, Neuvogen, Inc., and Eureka Therapeutics; patent holder with Tmunity, Inc., Stemline, Inc., and Novartis Pharma; grant/ research support from Servier Pharmaceuticals; and inventor of the H3.3K27M TCR, IL-13Ra2 (345-353:1A9V) peptide, and EGFRvIII-CAR, for which exclusive licensing agreements have been executed with Tmunity, Inc., Stemline, Inc., and Novartis Pharma, respectively.

\section{Author Contributions}

Conception and design: Haddad. Drafting the article: Haddad, Young, Oh. Critically revising the article: all authors. Reviewed submitted version of manuscript: Aghi, Haddad, Young, Okada. Study supervision: Okada.

\section{Correspondence}

Manish K. Aghi: University of California, San Francisco, CA. manish.aghi@ucsf.edu. 